\title{
On the Analysis of Non-homogeneous Laminates Using the Refined Zigzag Theory
}

\author{
Fernando G. Flores $^{a}$, Sergio Oller ${ }^{b, c}$, Liz G. Nallim $^{d}$ \\ ${ }^{a}$ IDIT-Departamento de Estructuras, Universidad Nacional de Córdoba and CONICET, \\ Av. Velez Sarsfield 1611, 5016 Córdoba-Argentina, fernando.flores@unc.edu.ar, \\ te: 54-351-5353800 int 721 \\ ${ }^{b}$ CIMNE International Center for Numerical Method Engineering, Spain \\ c UPC, Technical University of Catalonia (Barcelona Tech), Edif. C1, Campus Nord, \\ Jordi Girona 1-3, 08034 Barcelona, Spain \\ ${ }^{d}$ Facultad de Ingeniería, INIQUI (CONICET), Universidad Nacional de Salta, Av. \\ Bolivia 5150, 4400 Salta, Argentina
}

\begin{abstract}
This work shows possibilities and limitations of the refined zigzag theory (RZT) that has been used in different structural (beam, plate and shell) finite elements. The refined zigzag theory can deal with composite laminates, adding only one nodal degree of freedom per spatial dimension of the laminate, obtaining very good accuracy. It assumes that the in-plane displacements have a piece-wise linear shape across the thickness depending on the shear stiffness of each composite layer. This paper presents the main aspects of a beam/shell of revolution element used for the numerical simulations. The details of the refined zigzag theory are given also in order to discuss some limitationss that occur when dealing with the non-linear phenomenon of delamination. Two examples are presented and discussed, including different inhomogeneities that show the limitations of the RZT for the treatment of partially delaminated beams.
\end{abstract}

Keywords: Composite Laminates, Refined zigzag theory, Finite Elements, Delamination

\section{Introduction}

The refined zigzag theory(RZT), oriented to the treatment of composite laminates, is an evolution of the zigzag theories proposed from the 80s (an historical review of these theories can be seen in [2]). The RZT [18]) enhaces the first order shear deformation theory (FSDT), that includes 5 degrees of 
freedom (three displacements of the middle surface of the shell plus the two in-plane components of the normal rotation) and the hypothesis of linear inplane displacements across the thickness, adding only two degrees of freedom corresponding to the amplitudes of hierarchical in-plane displacements over the linear approach. This theory leads to constant transverse shear stresses in each layer (and therefore discontinuous in the thickness of the laminate), but allows to treat clamped boundary conditions that was a limitation presented by the initial zigzag theories. The transverse shear stresses computed directly from shear strains and using the constitutive relationship at each point of the thickness show in many cases a poor approximation. An accurate recovery of the shear stresses requires the integration across the laminate thickness of the in-plane equilibrium equations of the beam or shell, that involve the computation of stress derivatives between finite elements. To avoid this, mixed versions of the RZT have been developed $([13,17])$ where other improvements in the definition of additional displacements were also included. The RZT has been implemented, in all cases with linear kinematics, in beam finite elements ([10, 15, 3, 14]), in flat plate elements ([18, 4, 12, 21, 1]) and in double curvature elements ([20]). It has also been implemented including non-linear kinematics restricted to small elastic strains in double-curved shell elements ([8]) and in a solid-shell element ([9]). For homogeneous laminates, i.e. whose topology is maintained throughout the domain, the published results show a very good approximation to in-plane axial stresses in different types of sections but mainly of the sandwich type. This allows to obtain the transverse shear stresses by integration in the transverse direction of the in-plane equilibrium equations. Naturally a very good approximation to the axial stresses is associated with an even better approximation to the in-plane displacements. In general, the approximations do not include variation across the thickness of the normal displacement except in Reference [1] and collaterally in Reference [9]. Besides that, in several of the works cited, a very good approximation to frequencies and vibration modes in beams and flat plates has been reported. The use of non-linear kinematics has allowed to compare, in a few cases, buckling loads with very good accuracy. Another interesting aspect is the possibility of using this theory (RZT) for delamination problems. The difficulties that appear then are the lack of homogeneity and the need to update the transverse interpolation function during the delamination process $([5,6])$. More recently Groh and Tessler[11] evaluate the behavior of a partially delaminated beam (without following the delamination process) using the mixed version of the RZT.

In the present work an implementation of the RZT in a beam/shell of revolution finite element is shown. Initially, the shell theory used and the basic formulation (FSDT) of the beam/shell element are summarized. Next, 
two variants of the RZT are introduced, how it is implemented in the described element, and how the formulation of the element is modified for the case in which there are more than one defined sections. Then numerical simulations show a good correlation with comparisons with solid models and the limitations that appears when trying to simulate discontinuities due to delaminations. Finally, some conclusions are summarized.

\section{Standard beam/shell finite element (FSDT)}

\subsection{Summary of the shell theory}

The shell theory considered is a restriction to the two-dimensional case of the three-dimensional shell theory developed by Simo et al. ([16]). The configuration of the shell in $\mathbf{R}^{2}$ is defined by (see Figure 1):

a) the middle surface $\varphi$ defined by the mapping

$$
\varphi: \overline{\mathcal{S}} \longrightarrow \mathbf{R}^{2}
$$

b) the director field $\mathbf{t}$ defined by the mapping

$$
\mathbf{t}: \overline{\mathcal{S}} \longrightarrow S^{2} .
$$

The vector $\mathbf{t}$ defines the direction of a fiber across the thickness that remains straight during the deformation (generalized Kirchhoff hypothesis). The domain $\mathcal{S} \subset \mathbf{R}$ is supposed compact with points characterized by $\xi \subset \overline{\mathcal{S}}$.

With these notation, the geometry of the shell can be written as

$$
\boldsymbol{\Phi}:=\left\{\mathbf{x} \in \mathbf{R}^{2} / \mathbf{x}=\boldsymbol{\varphi}+z \mathbf{t}, \quad z \in[-h,+h]\right\}
$$

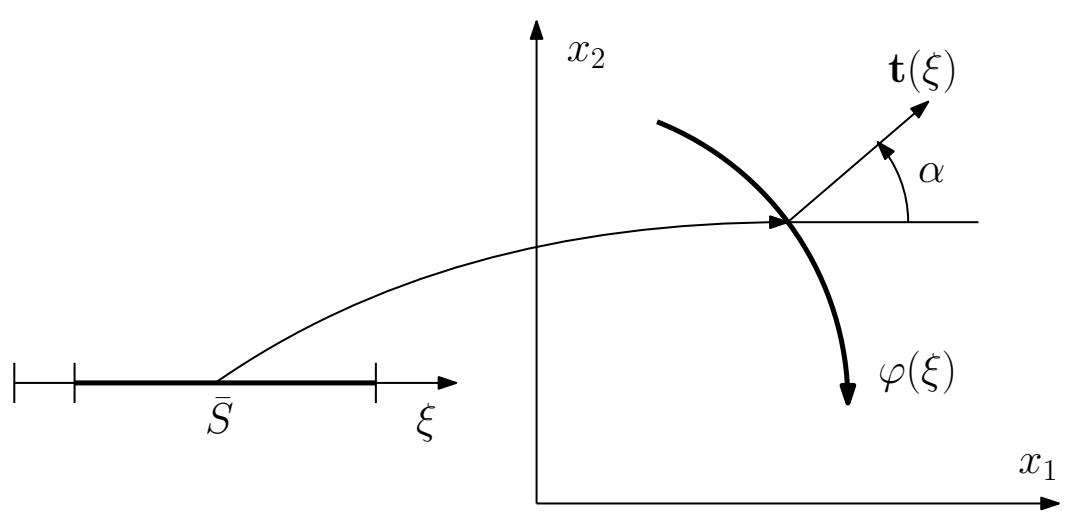

Figure 1: Basic definition of the geometry 
where $[-h,+h]$ defines the shell thickness. Using a Cartesian basis $\left\{\mathbf{e}_{1}, \mathbf{e}_{2}\right\}$ in $\mathbf{R}^{2}$ we can write

$$
\begin{gathered}
\boldsymbol{\varphi}=\boldsymbol{\varphi}^{i} \mathbf{e}_{i} \\
\boldsymbol{\varphi}^{\prime} \xi=\boldsymbol{\varphi}^{i} \mathbf{e}_{i} \\
\mathbf{t}=t^{i} \mathbf{e}_{i}
\end{gathered}
$$

Denoting geometrical variables in the original configuration $\Phi^{0}$ by ()$^{0}$ and defining a convective system on both the original and deformed configurations:

$$
\begin{gathered}
\left\{\boldsymbol{\varphi}_{\prime}^{0}, \mathbf{t}^{0}\right\} \equiv\left\{\mathbf{a}_{1}^{(0)}, \mathbf{a}_{2}^{(0)}\right\} \\
\left\{\boldsymbol{\varphi}^{\prime}, \mathbf{t}\right\} \equiv\left\{\mathbf{a}_{1}, \mathbf{a}_{2}\right\}
\end{gathered}
$$

where the coordinate $\xi$ is associated with convective direction 1 . The following surface measures can be defined over the middle surface

$$
\begin{aligned}
d \mu^{0} & =\bar{\jmath}_{0} d \xi d \zeta \\
d \mu & =\bar{\jmath} d \xi d \zeta
\end{aligned}
$$

with

$$
\begin{gathered}
\bar{\jmath}^{0}=\left(\boldsymbol{\varphi}_{\xi \xi}^{(0)} \times \mathbf{t}^{(0)}\right) \cdot \boldsymbol{\varphi}_{\zeta \zeta}^{(0)} \\
\bar{\jmath}=\left(\boldsymbol{\varphi}_{\xi}^{\prime} \times \mathbf{t}\right) \cdot \boldsymbol{\varphi}_{\zeta}^{\prime}
\end{gathered}
$$

and $\zeta$ the coordinate in the out-of-paper direction, that for the usual $2 \mathrm{D}$ cases, i.e. plane stress, plane strain or shell of revolution leads to $\varphi_{\zeta \zeta}=\mathbf{e}_{3}$. It is useful to define also:

$$
\bar{J}=\bar{\jmath} / \bar{\jmath}^{0}
$$

The deformation gradient at the middle surface $(z=0)$ that can be written as

$$
\overline{\mathbf{F}}:=\boldsymbol{\varphi}_{\prime_{\xi}} \otimes \mathbf{a}^{1(0)}+\mathbf{t} \otimes \mathbf{a}^{2(0)}+\boldsymbol{\varphi}_{\boldsymbol{\zeta}^{\prime}} \otimes \mathbf{e}^{3}
$$

allows to compute the Lagrange strains $\mathbf{E}=\frac{1}{2}\left(\mathbf{F}^{T} \mathbf{F}-\mathbf{1}\right)$ leading to

$$
\begin{aligned}
& \varepsilon_{1}=\frac{1}{2}\left[\boldsymbol{\varphi}_{\prime}^{\prime \xi} \cdot \boldsymbol{\varphi}_{\prime}^{\prime}-\boldsymbol{\varphi}_{\prime \xi}^{(0)} \cdot \boldsymbol{\varphi}_{\prime \xi}^{(0)}\right] \\
& \gamma=\varphi_{\prime} \cdot \mathbf{t}-\varphi_{\prime \xi}^{(0)} \cdot \mathbf{t}^{(0)} \\
& \chi_{1}=\varphi_{\prime \xi} \cdot \mathbf{t}^{\prime} \xi-\varphi_{\prime \xi}^{(0)} \cdot \mathbf{t}_{{ }^{\prime} \xi}^{(0)}
\end{aligned}
$$

For shells of revolution, the axial strain along the parallel and a second principal curvature must also be considered

$$
\varepsilon_{3}=\frac{1}{2}\left[\left(\frac{x_{1}}{x_{1}^{(0)}}\right)^{2}-1\right]
$$




$$
\chi_{3}=\frac{\sin \alpha^{(0)}}{x_{1}^{(0)}}-\frac{x_{1} \sin \alpha}{\left(x_{1}^{(0)}\right)^{2}}
$$

The stress resultants can be computed in terms of stress measures defined on the present or the original configurations

$$
\begin{aligned}
\mathbf{n}^{1} & =\frac{1}{\bar{\jmath}} \int_{-h}^{+h} \boldsymbol{\sigma} \mathbf{g}^{1} j d z \\
& =\frac{1}{\bar{\jmath}} \int_{-h}^{+h} \mathbf{P} \mathbf{g}^{1(0)} j^{0} d z \\
\mathbf{m}^{1}= & \mathbf{t} \times \frac{1}{\bar{\jmath}} \int_{-h}^{+h} z \boldsymbol{\sigma} \mathbf{g}^{1} j d z \\
= & \mathbf{t} \times \frac{1}{\bar{\jmath}} \int_{-h}^{+h} z \mathbf{P} \mathbf{g}^{1(0)} j^{0} d z=\mathbf{t} \times \tilde{\mathbf{m}}^{1} \\
\mathbf{l} & =\frac{1}{\bar{\jmath}} \int_{-h}^{+h} \boldsymbol{\sigma} \mathbf{g}^{2} j d z \\
& =\frac{1}{\bar{\jmath}} \int_{-h}^{+h} \mathbf{P} \mathbf{g}^{2(0)} j^{0} d z
\end{aligned}
$$

where $\mathbf{g}_{1}=\partial \mathbf{x} / \partial \xi, \quad \mathbf{g}_{2}=\partial \mathbf{x} / \partial z$, and $\mathbf{g}^{1}, \mathbf{g}^{2}$ are the associated contravariant vectors. $\boldsymbol{\sigma}$ and $\mathbf{P}$ are the Cauchy and first Piola-Kirchhoff stress tensors respectively. Besides $\mathbf{n}^{1}$ and $\mathbf{m}^{1}$ are the resultant stress and bending moment along the line $\xi=$ cte, while $\mathbf{l}$ is the across-the-thickness stress resultant. The vector $\tilde{\mathbf{m}}^{1}$ is denoted "director bending moment" and allows to define the following "effective stress resultant"

$$
\begin{aligned}
& \tilde{n}^{1}=n^{1}-\lambda_{1} \tilde{m}^{1} \\
& \tilde{q}=n^{13}-\lambda_{1} \tilde{m}^{1}
\end{aligned}
$$

where $\lambda_{i}$ are obtained from the relation

$$
\mathbf{t}^{\prime} 1=\lambda_{1} \varphi_{\prime 1}+\lambda_{2} \mathbf{t}
$$

For shells of revolution the following stress resultants must also be considered

$$
\begin{aligned}
\tilde{n}^{3} & =\frac{1}{\bar{j}} \int_{-h}^{+h} \mathbf{e}^{3} \boldsymbol{\sigma} \mathbf{e}^{3} j d z \\
& =\frac{1}{\bar{j}} \int_{-h}^{+h} \mathbf{e}^{3} \mathbf{P} \mathbf{e}^{3} j^{0} d z \\
\tilde{m}^{3} & =\frac{1}{\bar{j}} \int_{-h}^{+h} z \mathbf{e}^{3} \boldsymbol{\sigma} \mathbf{e}^{3} j d z \\
& =\frac{1}{\bar{j}} \int_{-h}^{+h} z \mathbf{e}^{3} \mathbf{P} \mathbf{e}^{3} j^{0} d z
\end{aligned}
$$


Using the expressions of internal power per unit length, it can be shown that the effective stress resultants defined above (18-21) are conjugated of the generalized Lagrangian strains (10-14).

$$
\begin{aligned}
\dot{w} & =\int_{A} \mathbf{P}: \dot{\mathbf{F}} d A \\
& =\int_{A}\left[\tilde{n}^{\alpha \alpha} \dot{\varepsilon}_{\alpha \alpha}+\tilde{q} \dot{\gamma}+\tilde{m}^{\alpha \alpha} \dot{\kappa}_{\alpha \alpha}\right] d \mu
\end{aligned}
$$

Assuming the existence of an internal energy function $w$, resorting to the Clausius-Duhem inequality and following standard arguments, hyperelastic constitutive equations can be formulated in the form

$$
\begin{aligned}
\tilde{n}^{\alpha} & =\bar{\rho} \frac{\partial w}{\partial \varepsilon_{\alpha}} \\
\tilde{q} & =\bar{\rho} \frac{\partial w}{\partial \gamma} \\
\tilde{m}^{\alpha} & =\bar{\rho} \frac{\partial w}{\partial \chi_{\alpha}}
\end{aligned}
$$

with

$$
\bar{\rho}=\frac{1}{\bar{\jmath}} \int_{-h}^{+h} \rho j d \xi
$$

\subsection{Implemented Finite Elements}

The beam/shell model defined above, that implies $C^{0}$ continuity and that can be associated with a first order shear deformation theory (FSDT), was implemented in a simple isoparametric finite element [7], of two and three nodes with three degrees of freedom per node (both in-plane displacements and the rotation of the director). To avoid transverse shear locking reduced integration is used, with one integration point for the 2-node element and two integration points for the quadratic 3-node element that can discretize curved surfaces in more detail.

\section{The Refined Zigzag Theory RZT and the RZT}

The refined zigzag theory (RZT), proposed and developed by Tessler and coworkers [18], has been implemented in multiple structural finite elements. The objective is to substantially improve the FSDT when laminated sections are considered, particularly sandwich sections, i.e. those composed of two stiff external layers and a flexible core.

In the FSDT the transverse shear strain $\gamma(z)$ is constant across the thickness therefore the shear stress $\tau(z)$ is discontinuous between two layers with different shear modulus. To reduce this discontinuity the transverse shear 
strains are modified including (hierarchical) additional in-plane displacements $\psi$ with a zigzag transverse profile $\phi(z)$ that has constant derivative $\beta^{k}=\phi_{\prime_{z}}^{k}$ at each layer $k$ :

$$
u_{1}(z)=u_{1}^{F S D T}(z)+\psi \phi(z)
$$

The total shear strain can now be seem as the sum of two components: one constant $\eta$ and one discontinuous that lead to two transverse shear stress components: a discontinuous one and a constant one respectively. The constant strain component $\eta$ can be seen as a modification of the constant strain component of the Reissner-Mindling plate theory

$$
\eta=\gamma-\psi
$$

which as before leads to a discontinuity of transverse shear stress between layers. The definition of the profile function $\phi(z)$ from the shear modules of each layer $G^{k}(k=1 . . N$ with $N$ the number of layers $)$ is based on the idea that shear stresses are written as the sum of two parts

$$
\begin{aligned}
\tau^{k}(z) & =G^{k} \eta+G^{k}\left(1+\phi_{\prime z}^{k}\right) \psi \\
& =G^{k} \eta+G^{k}\left[\left(1+\beta^{k}\right)\right] \psi \\
& =G^{k} \eta+\bar{\tau}^{k}(z)
\end{aligned}
$$

and to enforce the continuity of the component $\bar{\tau}^{k}(z)$ at each interface between layers (there are $N-1$ interfaces), that leads to

$$
\begin{aligned}
G^{k}\left[\left(1+\beta^{k}\right)\right] & =\bar{G} \\
\beta^{k} & =\frac{\bar{G}}{G^{k}}-1
\end{aligned}
$$

where $\bar{G}$ results from the condition $\int_{-h}^{+h} \beta d z=0$ (with $2 h$ the total thickness and $h^{k}$ the thickness of each layer)

$$
\int_{-h}^{+h} \beta^{k} d z=\sum_{k=1}^{N}\left(\frac{\bar{G}}{G^{k}}-1\right) h_{k}=\bar{G} \sum_{k=1}^{N} \frac{h_{k}}{G^{k}}-2 h=0
$$

then

$$
\bar{G}=\frac{2 h}{\sum_{k=1}^{N} \frac{h_{k}}{G^{k}}}
$$

This original version of the RZT leads to a stress $\tau(z)$ that is the sum of a discontinuous term (associated with $\eta$ ) and a constant term $\bar{\tau}$, which makes 


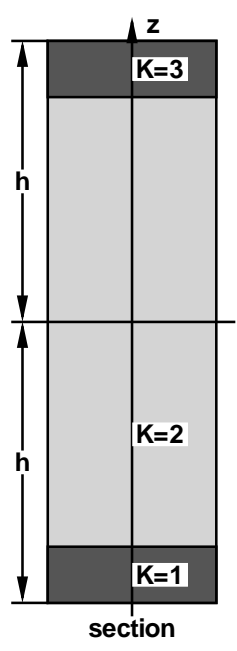

(a)

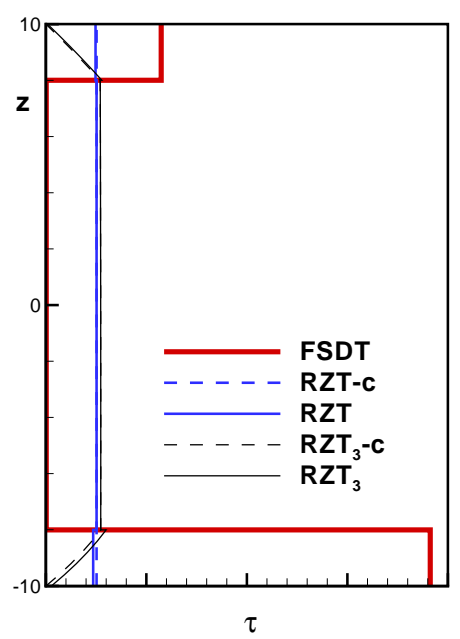

(b)

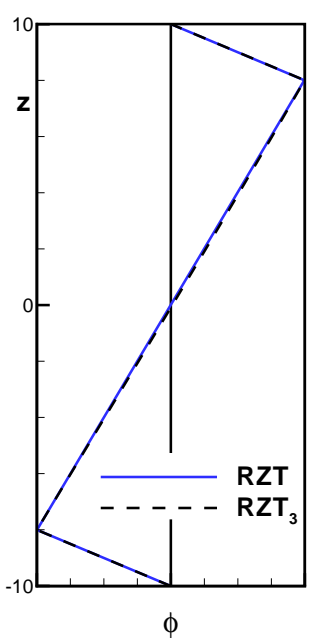

(c)

Figure 2: (a)Section, (b)Profile of $\tau$ and (c)function $\phi$ in FSDT, RZT and RZT $_{3}$

it impossible to simultaneously satisfy continuity and boundary conditions. The achievement of the RZT is to go from a FSDT with constant $\gamma(z)$ and where the shearing force is taken only by the stiff layers (in a sandwich section) to a major contribution of the core to support the shearing force due to the component $\bar{\tau}$. The main advantage of the RZT is that the profile $\phi(z)$ makes it possible to better fit the axial stresses $\sigma_{x}(z)$. This leads to a more realistic bending stiffness, which in turn allows to obtain a transverse shear stress profile with very good accuracy if it is computed by integration across the thickness of the in-plane equilibrium equations and not through the usual way of kinematics relations plus constitutive equations.

The-Figure 2 shows what happens in an asymmetrical sandwich section with a very flexible core and a bottom layer with a transverse modulus of elasticity three times that of the top layer. The example corresponds to a cantilever beam with a constant shearing force (see Figure 3 and Table 1). The stress profile corresponds to half length where the influence of the boundary conditions is quite low. For the FSDT, where the profile shows strong discontinuities, all the shearing force is held in equilibrium by the stiff layers proportionally to each shear modulus $G^{k}$. Two profiles are shown for the RZT, one corresponding to the continuos part $\bar{\tau}$ (RZT-c) and the total one (RZT). Clearly it does no satisfies the null stress boundary conditions at the external surfaces but in contrast with the FSDT the core contributes substantially to equilibrate the shearing force.

A very interesting aspect presented in [13] is the definition of a new zigzag 
function. In that work the continuous part $\bar{\tau}^{k}(z)$ is improved such that besides being continuous is variable and nullifies at the external surfaces. In that way if $\eta \cong 0$ a more accurate approximation is obtained and, for sections far from boundary restrictions, a direct computation (i.e. without integration of equilibrium equation) of the shear stresses can be found reliable.

Obtaining the new function $\phi(z)$, which we will denote as $\mathrm{RZT}_{3}$, begins from proposing for the additional displacement the form:

$$
u_{a}(z)=\left[z^{2} \chi_{0}+z^{3} \omega_{0}+v^{k}(z)\right] \psi=\phi(z) \psi
$$

where $v(z)$ is piece-wise linear (zigzag), and is just a part of the new $\phi(z)$. Two terms than influences all the thickness have been added: one quadratic $z^{2} \chi_{0}$ and the other cubic $z^{3} \omega_{0}$. The shear stresses are written in the same form as in the original RZT (with $\eta=\gamma-\psi$ )

$$
\begin{aligned}
\tau^{k}(z) & =G^{k} \eta+G^{k}\left[\left(1+v_{z}\right) \psi+2 z \chi_{0} \psi+3 z^{2} \omega_{0} \psi\right] \\
& =G^{k} \eta+\bar{\tau}^{k}(z)
\end{aligned}
$$

To redefine the zigzag function $\phi(z)$ the following additional conditions are enforced: at the external surfaces is asked ( 2 new conditions with respect to the original version)

$$
\bar{\tau}^{k}(z= \pm h)=0
$$

(Note that component $\bar{\tau}^{k}(z)$ is nullified, but the total shear stress there $\tau^{k}(z= \pm h)=G^{1-N} \eta$ is only zero if $\left.\eta=0\right)$ then

$$
\begin{aligned}
& \left(1+v_{\prime_{z}}^{N}\right)+2 h \chi_{0}+3 h^{2} \omega_{0}=0 \\
& \left(1+v_{\prime_{z}}^{1}\right)-2 h \chi_{0}+3 h^{2} \omega_{0}=0
\end{aligned}
$$

alternatively adding and subtracting, two uncoupled expressions are obtained for $\chi_{0}$ and $\omega_{0}$

$$
\begin{array}{r}
\left(2+v_{\prime_{z}}^{N}+v_{\prime_{z}}^{1}\right)+6 h^{2} \omega_{0}=0 \\
\left(v_{\prime_{z}}^{N}-+v_{\prime_{z}}^{1}\right)+4 h \chi_{0}=0
\end{array}
$$

solving

$$
\begin{aligned}
& \chi_{0}=-\frac{\left(v_{\prime z}^{N}-v_{\iota_{z}}^{1}\right)}{4 h} \\
& \omega_{0}=-\frac{\left(2+v_{\prime_{z}}^{N}+v_{\prime_{z}}^{1}\right)}{6 h^{2}}
\end{aligned}
$$


Introducing this new definition of the additional displacements in (25) the shear stress is written as:

$$
\begin{aligned}
\tau^{k}(z) & =G^{k} \eta+G^{k}\left[\left(1+v_{\prime}\right)-2 z \chi_{0}-3 z^{2} \omega_{0}\right] \psi \\
& =G^{k} \eta+\bar{\tau}^{k}(z)
\end{aligned}
$$

Similarly to the original RZT at each interface $(N-1$ conditions) the continuity of component $\bar{\tau}$ is imposed

$$
\begin{aligned}
\bar{\tau}^{k}\left(z_{k}\right)-\bar{\tau}^{k+1}\left(z_{k}\right) & =0 \\
G^{k}\left[\left(1+v_{\prime_{z}}^{k}\right)+2 z_{k} \chi_{0}+3 z_{k}^{2} \omega_{0}\right]-G^{k+1}\left[\left(1+v_{\prime z}^{k+1}\right)+2 z_{k} \chi_{0}+3 z_{k}^{2} \omega_{0}\right] & =0 \\
\frac{v_{\prime z}^{k}}{1-G^{k+1} / G^{k}}+\frac{v_{\prime z}^{k+1}}{1-G^{k} / G^{k+1}}+2 z_{k} \chi_{0}+3 z_{k}^{2} \omega_{0}+1 & =0
\end{aligned}
$$

Note that this condition of continuity can not be enforced in this way if the shear modulus of the adjacent layers is the same. In that case the condition can be written simple as

$$
v_{\prime_{z}}^{k}-v_{\prime_{z}}^{k+1}=0
$$

Finally the condition that the additional displacement is zero at each external surface (with $v_{0}=v(z=-h)$ )

$$
\begin{gathered}
\phi(z=-h)=\phi(z=h)=0 \\
\phi(z=-h)=h^{2} \chi_{0}-h^{3} \omega_{0}+v_{0} \\
\phi(z=h)=h^{2} \chi_{0}+h^{3} \omega_{0}+v_{0}
\end{gathered}
$$

Thus $N+3$ conditions are enforced, where the unknowns are $\chi_{0}, \omega_{0}$, the $v_{\prime z}^{k}(k=1 . . N)$ and $v_{0}$.

The Figure 2.b also shows the shear stress profile using this approach. It can be seen that in the stiff external layers the stress begins practically from zero at the external surfaces ( $\eta$ is very low at the second part of the beam) and grows until a value that is practically constant in the core. Figure 2.d shows the functions $\phi(z)$ for both versions of the RZT. Although they look as the same function, the quadratic and cubic terms allow to adjust the null stress conditions at the external surfaces. Additionally, note that the $\phi(z)$ of the original RZT is null for one layer sections (one single material) or when there is no change in the value of $G^{k}$ between layers, as can be deduced from the expressions (28) and (30). For such cases a special technique has been proposed in [19] to obtain a non-zero $\phi(z)$. In contrast the $\mathrm{RZT}_{3}$ leads for one material sections to a parabolic variation of the continuous $\bar{\tau}$ without resorting to any special technique. 


\section{Implementation of the $\mathrm{RZT}_{3}$ in a beam/shell element}

In the convective system the configuration (3) can now be written as:

$$
\mathbf{\Phi}:=\left\{\mathbf{x} \in \mathbf{R}^{2} / \mathbf{x}=\varphi+z \mathbf{t}+\psi \phi(z) \mathbf{a}_{1}, \quad z \in[-h,+h]\right\}
$$

and, in order to keep the implementation simple, small strains are assumed, in such a way that the contributions of the base element (FSDT) can be added directly with those resulting from the additional displacements $\left(\psi \phi(z) \mathbf{a}_{1}\right)$.

4.1. Model with a geometrically and mechanically constant section in the beam span

The strains involved are

$$
\begin{aligned}
\varepsilon_{1}^{k} & =\left[1, z, \phi^{k}\right]\left[\begin{array}{c}
\varepsilon_{1} \\
\chi_{1} \\
\psi^{\prime} 1
\end{array}\right]=\mathbf{S}_{p} \hat{\varepsilon}_{p} \\
\gamma_{1 z}^{k} & =\left[1, \beta^{k}\right]\left[\begin{array}{c}
\gamma \\
\psi
\end{array}\right]=\mathbf{S}_{t} \hat{\varepsilon}_{t}
\end{aligned}
$$

The internal strain energy per unit length of the beam, associated with the stresses in the cross section normal to $\mathbf{a}_{1}$, results from integrating in the area of the cross section:

$$
\begin{aligned}
& w_{1}=\frac{1}{2} \int_{A}\left(\varepsilon_{1}^{k} \sigma_{1}^{k}+\gamma_{1 z}^{k} \tau_{1 z}^{k}\right) d A \\
& =\frac{1}{2} \int_{A}\left(\hat{\varepsilon}_{p}^{T} \mathbf{S}_{p}^{T} E^{k} \mathbf{S}_{p} \hat{\boldsymbol{\varepsilon}}_{p}+\hat{\boldsymbol{\varepsilon}}_{t}^{T} \mathbf{S}_{t}^{T} G^{k} \mathbf{S}_{t} \hat{\boldsymbol{\varepsilon}}_{t}\right) d A \\
& =\frac{1}{2} \int_{A}\left(\hat{\varepsilon}_{p}^{T}\left[\begin{array}{c}
1 \\
z \\
\phi^{k}
\end{array}\right] E^{k}\left[1, z, \phi^{k}\right] \hat{\varepsilon}_{p}+\hat{\varepsilon}_{t}^{T}\left[\begin{array}{c}
1 \\
\beta^{k}
\end{array}\right] G^{k}\left[1, \beta^{k}\right] \hat{\varepsilon}_{t}\right) d A \\
& =\frac{1}{2} \int_{A}\left(\hat{\varepsilon}_{p}^{T} E^{k}\left[\begin{array}{ccc}
1 & z & \phi^{k} \\
z & z^{2} & z \phi^{k} \\
\phi^{k} & z \phi^{k} & \left(\phi^{k}\right)^{2}
\end{array}\right] \hat{\boldsymbol{\varepsilon}}_{p}+\hat{\boldsymbol{\varepsilon}}_{t}^{T} G^{k}\left[\begin{array}{cc}
1 & \beta^{k} \\
\beta^{k} & \left(\beta^{k}\right)^{2}
\end{array}\right] \hat{\boldsymbol{\varepsilon}}_{t}\right) d A
\end{aligned}
$$

Defining

$$
\mathbf{D}_{p}=\int_{A} E^{k}\left[\begin{array}{ccc}
1 & z & \phi^{k} \\
z & z^{2} & z \phi^{k} \\
\phi^{k} & z \phi^{k} & \left(\phi^{k}\right)^{2}
\end{array}\right] d A
$$

and

$$
\mathbf{D}_{s}=\int_{A} G^{k}\left[\begin{array}{cc}
1 & \beta^{k} \\
\beta^{k} & \left(\beta^{k}\right)^{2}
\end{array}\right] d A
$$


Thus the expression of the internal strain energy can be written

$$
w_{1}=\frac{1}{2}\left(\hat{\varepsilon}_{p}^{T} \mathbf{D}_{p} \hat{\varepsilon}_{p}+\hat{\varepsilon}_{t}^{T} \mathbf{D}_{t} \hat{\varepsilon}_{t}\right)
$$

that allows to define the stress resultants

$$
\hat{\boldsymbol{\sigma}}=\left[\begin{array}{c}
\hat{\boldsymbol{\sigma}}_{p} \\
\hat{\boldsymbol{\sigma}}_{t}
\end{array}\right]=\left[\begin{array}{cc}
\mathbf{D}_{p} & \mathbf{0} \\
\mathbf{0} & \mathbf{D}_{t}
\end{array}\right]\left[\begin{array}{c}
\hat{\boldsymbol{\varepsilon}}_{p} \\
\hat{\boldsymbol{\varepsilon}}_{t}
\end{array}\right]
$$

\subsection{Finite element models for non-homogeneous laminates}

The implementation of the RZT is based on the idea of hierarchical degrees of freedom $\psi$ associated with shape functions $\phi$. That is to say that the nodal hierarchical degrees of freedom $\psi^{i}$ are on the one hand associated with nodal-shaped functions $N^{i}(\xi)$ in the plane of the laminate (or axial direction of the beam ) and on the other hand to the function $\phi(z)$ in the transverse direction. When a single laminate exists, i.e. when the section is the same along the beam or shell (homogeneous laminate), the function $\phi(z)$ is unique throughout the domain.

When the properties of the laminate change along the beam or shell, the function $\phi(z)$ is no longer the same for the entire model. Since the nodal unknowns are the amplitudes $\psi^{i}$ and they multiply to the $\phi(z)$, if the latter are not the same at both elements sharing the node, the finite element model is not conforming any longer, that is to say, the continuity is lost, since the displacements cease to be the same on both sides of the interface between elements. A "non-conforming" model, in this case, leads to inconsistent results.

It is then necessary to modify the transverse interpolation in order to maintain a continuous model. A case where the properties of the section change is when it presents some kind of damage. For example, to simulate the delamination of an interlaminar section, a scalar damage model has been used [5], which involves degrading the (longitudinal and transverse) modulus of elasticity of the material of a very thin layer. This change in the properties of a layer requires modifying the associated function $\phi(z)$.

A distinctive aspect of the FEM is that in each element $e$ there is a single "material" or a single "section" and as we have seen the function $\phi$ defined by the RZT depends on the properties of the materials. Then, it can be said, that each element has a function $\phi^{e}$ associated with the corresponding laminate. Then, to deal with this case, two conceptually simple options are proposed, which consist in supposing that in the interface between elements the function $\phi$ associated with node $n$ results from:

a) the average of the properties of the sections associated with the elements adjacent to the node $\phi^{n}=f(\bar{G})$. 
b) the average of the functions associated with the elements adjacent to the node

In this way it can be assumed that the in-plane additional displacements result from the interpolation (for a two-node element)

$$
u_{a}(\xi, z)=N^{1}(\xi) \phi^{1}(z) \psi^{1}+N^{2}(\xi) \phi^{2}(z) \psi^{2}
$$

where the $N^{n}(\xi)$ are the linear Lagrange polynomials

$$
\begin{aligned}
& N^{1}(\xi)=\frac{1}{2}(1-\xi) \\
& N^{2}(\xi)=\frac{1}{2}(1+\xi)
\end{aligned}
$$

whereas the transverse interpolation functions will be, for the case a)

$$
\left.\begin{array}{l}
\phi^{1}(z)=f\left(\frac{1}{2}\left[G^{l 1}(z)+G^{r 1}(z)\right]\right) \\
\phi^{2}(z)=f\left(\frac{1}{2}\left[G^{l 2}(z)+G^{r 2}(z)\right]\right.
\end{array}\right\}
$$

where $G^{l I}(z)$ and $G^{r I}(z)$ are the mechanical properties of the sections on the left $(l)$ and on the right $(r)$ of node $I$.

While for case b) they are

$$
\begin{aligned}
& \phi^{1}(z)=\frac{1}{2}\left(\phi^{l 1}(z)+\phi^{r 1}(z)\right) \\
& \phi^{2}(z)=\frac{1}{2}\left(\phi^{l 2}(z)+\phi^{r 2}(z)\right)
\end{aligned}
$$

with $\phi^{l I}$ and $\phi^{r I}$ the profile functions computed with the properties or the sections of element to the left and to the right of node $I$.

The additional axial strain results

$$
\varepsilon_{1 \phi}=-\frac{1}{L} \phi^{1}(z) \psi^{1}+\frac{1}{L} \phi^{2}(z) \psi^{2}
$$

While the additional shear strain at the element center is

$$
\gamma_{\phi}=\frac{\partial u_{a}}{\partial z}=\frac{1}{2} \beta^{1}(z) \psi^{1}+\frac{1}{2} \beta^{2}(z) \psi^{2}
$$

Then the total strains can be written as

$$
\begin{aligned}
\varepsilon_{1}^{k} & =\left[1, z, \phi^{1}, \phi^{2}\right]\left[\begin{array}{r}
\varepsilon_{1} \\
\chi_{1} \\
-\frac{1}{L} \psi^{1} \\
\frac{1}{L} \psi^{2}
\end{array}\right]=\mathbf{S}^{p} \overline{\boldsymbol{\varepsilon}}^{p} \\
\gamma_{1 z}^{k} & =\left[1, \beta^{1}, \beta^{2}\right]\left[\begin{array}{r}
\gamma \\
\frac{1}{2} \psi^{1} \\
\frac{1}{2} \psi^{2}
\end{array}\right]=\mathbf{S}^{t} \overline{\boldsymbol{\varepsilon}}^{t}
\end{aligned}
$$


Some differences appear with respect to the formulation with homogeneous section, since now, for practical implementation purposes, the contributions of each node, and therefore of each function of transverse interpolation $\phi$, are split.

The relevant stress components can be written in the form

$$
\begin{aligned}
\sigma_{1}^{k} & =E^{k} \varepsilon_{1}^{k}=E^{k} \mathbf{S}^{p} \bar{\varepsilon}^{p} \\
\tau_{1 z}^{k} & =G^{k} \gamma_{1 z}^{k}=G^{k} \mathbf{S}^{t} \bar{\varepsilon}^{t}
\end{aligned}
$$

That replaced into the internal strain energy per unit length, allows to integrate in the section (compare with eq. (49)):

$$
\begin{aligned}
w_{1} & =\frac{1}{2} \int_{A}\left(\varepsilon_{1}^{k} \sigma_{1}^{k}+\gamma_{1 z}^{k} \tau_{1 z}^{k}\right) d A \\
& =\frac{1}{2} \int_{A}\left(\bar{\varepsilon}^{p T} \mathbf{S}^{p T} E^{k} \mathbf{S}^{p} \overline{\boldsymbol{\varepsilon}}^{p}+\overline{\boldsymbol{\varepsilon}}^{t T} \mathbf{S}^{t T} G^{k} \mathbf{S}^{t} \overline{\boldsymbol{\varepsilon}}^{t}\right) d A \\
& =\frac{1}{2} \int_{A}\left(\overline{\boldsymbol{\varepsilon}}_{p}^{T}\left[\begin{array}{c}
1 \\
z \\
\phi^{1} \\
\phi^{2}
\end{array}\right] E^{k}\left[1, z, \phi^{1}, \phi^{2}\right] \overline{\boldsymbol{\varepsilon}}_{p}+\overline{\boldsymbol{\varepsilon}}_{t}^{T}\left[\begin{array}{c}
1 \\
\beta^{1} \\
\beta^{2}
\end{array}\right] G^{k}\left[1, \beta^{1}, \beta^{2}\right] \overline{\boldsymbol{\varepsilon}}_{t}\right) d A
\end{aligned}
$$

Then the expression of internal strain energy per unit length can be written as

$$
w_{1}=\frac{1}{2}\left(\bar{\varepsilon}^{p T} \mathbf{D}^{p} \bar{\varepsilon}^{p}+\bar{\varepsilon}^{t T} \mathbf{D}^{t} \bar{\varepsilon}^{t}\right)
$$

where the following matrices have been defined

$$
\begin{gathered}
\mathbf{D}^{p}=\int_{A} E^{k}\left[\begin{array}{cccc}
1 & z & \phi^{1} & \phi^{2} \\
z & z^{2} & z \phi^{1} & z \phi^{2} \\
\phi^{1} & z \phi^{1} & \left(\phi^{1}\right)^{2} & \phi^{1} \phi^{2} \\
\phi^{2} & z \phi^{2} & \phi^{1} \phi^{2} & \left(\phi^{2}\right)^{2}
\end{array}\right] d A \\
\mathbf{D}^{t}=\int_{A} G^{k}\left[\begin{array}{ccc}
1 & \beta^{1} & \beta^{2} \\
\beta^{1} & \left(\beta^{1}\right)^{2} & \beta^{1} \beta^{2} \\
\beta^{2} & \beta^{1} \beta^{2} & \left(\beta^{2}\right)^{2}
\end{array}\right] d A
\end{gathered}
$$

That allows to define the stress resultants

$$
\overline{\boldsymbol{\sigma}}=\left[\begin{array}{c}
\overline{\boldsymbol{\sigma}}^{p} \\
\overline{\boldsymbol{\sigma}}^{t}
\end{array}\right]=\left[\begin{array}{cc}
\mathbf{D}^{p} & \mathbf{0} \\
\mathbf{0} & \mathbf{D}^{t}
\end{array}\right]\left[\begin{array}{c}
\overline{\boldsymbol{\varepsilon}}^{p} \\
\overline{\boldsymbol{\varepsilon}}^{t}
\end{array}\right]
$$


where now

$$
\left[\begin{array}{c}
\overline{\boldsymbol{\sigma}}^{p} \\
\overline{\boldsymbol{\sigma}}^{t}
\end{array}\right]=\left[\begin{array}{c}
N \\
M \\
M_{\phi}^{1} \\
M_{\phi}^{2} \\
Q \\
Q_{\phi}^{1} \\
Q_{\phi}^{2}
\end{array}\right] \quad\left[\begin{array}{c}
\overline{\boldsymbol{\varepsilon}}^{p} \\
\overline{\boldsymbol{\varepsilon}}^{t}
\end{array}\right]=\left[\begin{array}{c}
\varepsilon_{1} \\
\chi_{1} \\
-\frac{1}{L} \psi^{1} \\
\frac{1}{L} \psi^{2} \\
\gamma \\
\frac{1}{2} \psi^{1} \\
\frac{1}{2} \psi^{2}
\end{array}\right]
$$

from which additional stress resultants are the average of the values in $\overline{\boldsymbol{\sigma}}$ and the associated strains result from adding the contributions in $\bar{\varepsilon}$;

$$
\left[\begin{array}{c}
\hat{\boldsymbol{\sigma}}_{p} \\
\hat{\boldsymbol{\sigma}}_{t}
\end{array}\right]=\left[\begin{array}{c}
N \\
M \\
\frac{1}{2} M_{\phi}^{1}+\frac{1}{2} M_{\phi}^{2} \\
Q \\
\frac{1}{2} Q_{\phi}^{1}+\frac{1}{2} Q_{\phi}^{2}
\end{array}\right] \quad\left[\begin{array}{c}
\hat{\boldsymbol{\varepsilon}}_{p} \\
\hat{\boldsymbol{\varepsilon}}_{t}
\end{array}\right]=\left[\begin{array}{c}
\varepsilon_{1} \\
\chi_{1} \\
\frac{1}{L}\left(\psi^{2}-\psi^{1}\right) \\
\gamma \\
\frac{1}{2}\left(\psi^{1}+\psi^{2}\right)
\end{array}\right]
$$

This proposal ensures continuity of the displacements, that is to say a $C^{0}$ conforming approach, but uses within a element a function $\phi$ that depends on the mechanical properties of the adjacent element. Individually in each element a function $\phi^{e}(z)$ is defined dependent on the mechanical properties of the section (which are a function of the level of damage in the case of delamination) that satisfies the continuity of the component $\bar{\tau}^{e}(z)$. But this property disappears when combining the functions of two elements with different properties (eq. 57) or using properties that are not strictly those of the element (eq. 56). This is what happens in the case of an element with contiguous elements (left and/or right) with different mechanical properties. It must then be pointed out that:

- That in such element $e$ the profile functions have to be combined using (56) or (57) that depend in a non-linear way on the properties of the material

- That the properties of the material associated with the element (and its level of damage) are kept

- And consequently that combination of $\phi$ between elements and the use of the element material will lead to a discontinuous $\bar{\tau}$ function and the properties used in its definition will be lost.

\section{Examples}

In the examples presented below, comparisons will be made between the $2 \mathrm{D}$ beam/shell element described in Section 2 (of two nodes B2) including the 
refined zigzag theory $\left(\mathrm{RZT}_{3}\right)$ and results obtained using a two-dimensional solid element (four-node quadrilateral Q4). The objective is to use the simplest possible configurations (one-dimensional in the case of the beam) so that the comparisons and the aspects that are intended to be shown are easy to visualize. We intend to compare two things: a) Overall structural behavior, including displacements, vibration frequency and buckling loads and b) Local stress states, including axial stress and shear stress profiles. On the other hand, we seek to evaluate the behavior of the RZT in case of an abrupt change in the properties of the section, which is what happens on the delamination front. Examples with some similar characteristics have been studied in Reference [11] to evaluate possibilities of the mixed RZT for the study of delamination. Here some of their conclusions will be contrasted with present results.

\subsection{Cantilever beam with constant shear}

The first example considered is a cantilever beam with a load at its free end, i.e. with a constant shearing force, as outlined in the Figure 3. A width $b=20 \mathrm{~mm}$ has been assumed. The section is an asymmetric sandwich composed of isotropic materials. The properties of the materials are indicated in the Table 1 where the thickness of each layer and the corresponding material are also indicated. Two sections are described, in the second case a thin layer $(0.01 \mathrm{~mm})$ in the union between the core and the upper layer is replaced by a damaged material (2d) with very low rigidity in order to simulate a total or partial delamination.

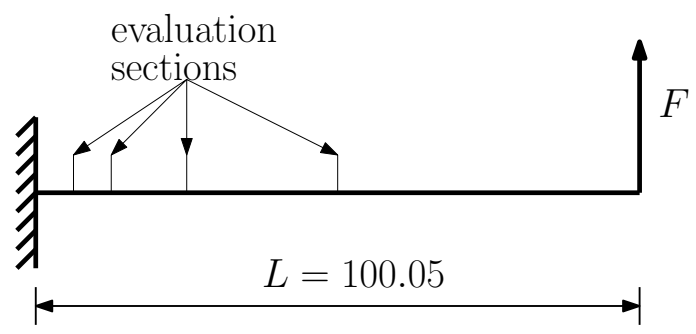

Figure 3: Analyzed beam

For the comparison a load $F=20.01 \mathrm{~N}$ is considered, that is a shearing force per unit width $T=1.0005 \mathrm{kN} / \mathrm{m}$ and an average shear stress over the cross-section of $\tau_{\text {ave }}=0.05 \mathrm{MPa}$. As reference a model with 4 -noded solid elements (Q4) was used, including 400 divisions along the beam span and 37 across the thickness, 12 in each external layer and 12 in $16 \mathrm{~mm}$ of the thickness of the core plus 1 element in the sublayer of thickness $0.01 \mathrm{~mm}$. To avoid an "ad-hoc" distribution of the load over the nodes at the free edge, the 


\begin{tabular}{|r|r|r|}
\hline Mat & $E[\mathrm{GPa}]$ & $G[\mathrm{GPa}]$ \\
\hline \hline 1 & 730 & 292 \\
\hline 2 & 0.73 & 0.292 \\
\hline $2 \mathrm{~d}$ & $0.73 \times 10^{-6}$ & $0.292 \times 10^{-6}$ \\
\hline 3 & 219 & 87.6 \\
\hline
\end{tabular}

(a)

\begin{tabular}{|c|r|}
\hline Mat & Thick. \\
\hline 3 & 2.00 \\
\hline 2 & 16.01 \\
\hline 1 & 2.00 \\
\hline
\end{tabular}

(b)

\begin{tabular}{|r|r|}
\hline Mat & Thick. \\
\hline 3 & 2.00 \\
\hline $2 \mathrm{~d}$ & 0.01 \\
\hline 2 & 16.00 \\
\hline 1 & 2.00 \\
\hline
\end{tabular}

(c)

Table 1: (a) Properties of the materials involved, (b) undamaged laminate and (c) damaged laminate

vertical displacements of those nodes are prescribed to have a single value. For the beam element (B2) a fine mesh of 400 elements was considered, with the same axial distribution as the mesh of solids, although a much coarser mesh leads to the same results. To compare the results obtained with both models, four sections have been selected, indicated in Figure 3, designated by $1-4$ and located respectively at $1: \frac{L}{16}, 2: \frac{L}{8}, 3: \frac{L}{4}$ and $4: \frac{L}{2}$ of the fixed support.

\subsubsection{Original undamaged beam}

For the beam model (B2) the vertical displacement of the center of the free edge results $u_{z}^{B 2}=0.01805 \mathrm{~mm}$ and for the solid model $u_{z}^{Q 4}=0.01808 \mathrm{~mm}$, with a difference of only $0.2 \%$. For a uniform density $\delta=4 \times 10^{3} \mathrm{~kg} / \mathrm{m}^{3}$ and maintaining the condition that all the nodes at the free edge move the same vertical value, the fundamental period obtained are $T^{B 2}=3.0982 \mathrm{~ms}$ and $T^{Q 4}=3.1042 \mathrm{~ms}$ respectively, also with a difference of $0.2 \%$ which is congruent with the slightly lower stiffness of the solid model.

The-Figure 4 shows the profiles of horizontal displacements and axial stresses for the four sections mentioned. The displacement profiles of the solid model are slightly curved in the zone closest to the support where some discrepancies with the beam model can be seen. These differences disappear as one moves away from the support. With respect to the axial stresses they are practically coincident between both models at the four sections analyzed. This is very important because it allows us to think that it is possible to evaluate shear stresses by integration in the z-direction of the axial equilibrium equation. Besides, the profiles closest to the support indicate a local bending of the lower layer with a sign change in the stresses, while in the more distant sections there are only tensile stresses in the lower layer.

The Figure 5.a shows the profiles of transverse shear stresses for the four mentioned sections obtained with the solid model and with the beam model by integration of the axial equilibrium equation using the computed axial stresses. The profile changes a lot in the first half of the beam then remains practically constant. In the vicinity of the support the shearing force is taken 

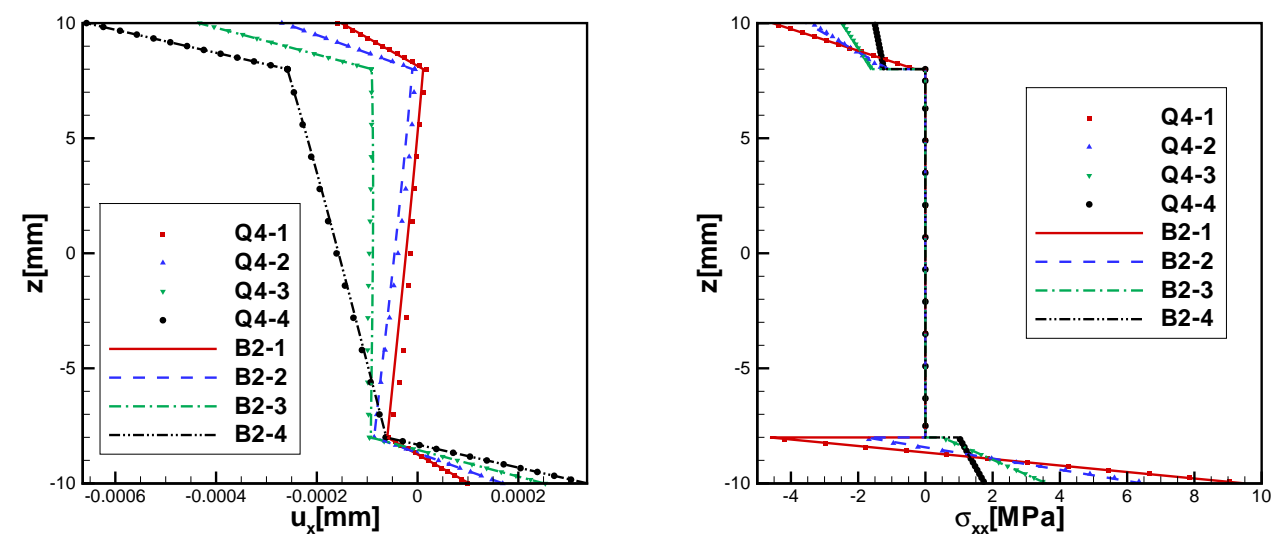

Figure 4: Profiles of horizontal displacements and axial stresses

mainly by the rigid layers, where the highest shear stresses $\tau_{x z}^{\max }$ occurs. Near the support the results are coincident in the core area but the reference model (Q4) indicates that the lower layer takes less shear than what the beam model indicates (and vice versa for the upper layer). An excellent agreement from $\frac{x}{L}=\frac{1}{8}$ on can be seen, and as one moves towards the free edge, the shearing force is mainly taken by the flexible core.

Figure 5.b shows the profiles of transverse shear stresses computed using the finite element standard based on displacements, that is, obtaining strains from the displacements and using the constitutive equations. In this figure there are two sets of curves, " $k+c$ " indicates kinematic plus constitutive and " $\mathrm{c}$ " is the continuous part of the $\mathrm{RZT}_{3}$, that is, without including the strain measure $\eta$. From this graph it can be concluded that:

- For all profiles, in the core zone the $\tau_{x z}$ computed by the $\mathrm{RZT}_{3}$ is almost independent of $\eta$ and coincide with the solid model

- The influence of $\eta$ decreases markedly when moving away from the fixed support and is almost null at half the beam.

- the total shear stress $(k+c)$ gives an average value over the outer layers but does not approximate correctly the variation in those layers.

It can be said that the warping restriction imposed by the fixed support is what precludes the flexible central part from contributing to support the shearing force. In the section closest to the support, the shearing force is taken by the rigid outer layers, where a parabolic variation of the shear 


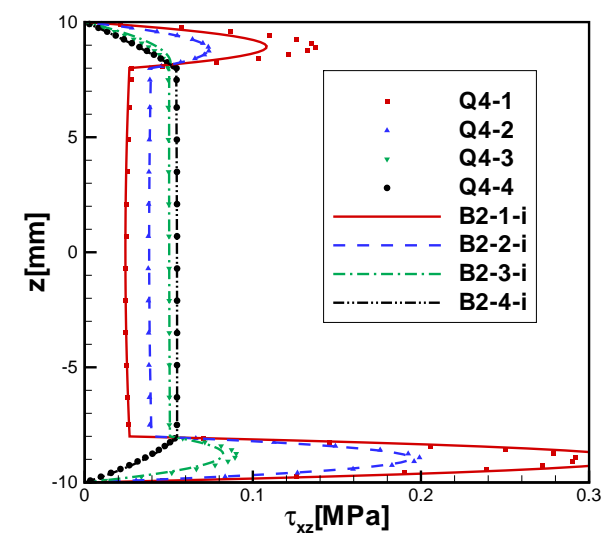

(a)

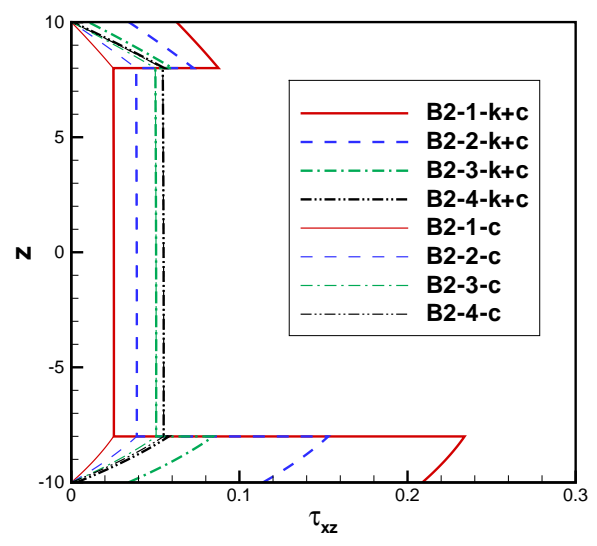

(b)

Figure 5: Profiles of shear stresses for the undamaged beam

stresses similar to what occurs in a section of homogeneous material is observed for both layers. For the sections away from the support, shear strains can develop in the central part.

For the case of the undamaged beam, an example with the same ratios between dimensions and between properties of the materials has been analyzed in Reference [11] with identical shear stress profiles.

\subsubsection{Damaged beam along its entire length}

In this case the vertical displacement of the center of the free edge results $u_{z}^{B 2}=0.18027 \mathrm{~mm}$ for the beam model and $u_{z}^{Q 4}=0.18794 \mathrm{~mm}$ for the solid model, with a difference above $4 \%$ that is explained below. The fundamental period now results $T^{B 2}=8.470 \mathrm{~ms}$ and $T^{Q 4}=8.704 \mathrm{~ms}$ and a difference of $2.7 \%$.

Figure 6 shows the profiles of horizontal displacements and axial stresses for the four sections mentioned. The displacement profiles are very similar in both models, slightly displaced to the left in the case of the beam model due to its larger stiffness. The RZT allows capturing the discontinuity in the weak layer, dividing the section into two parts where a linear approximation of the horizontal displacements seems appropriate. Regarding the axial stresses, although they look similar, there are some differences especially in the part closest to the support. This is important because it implies that when computing shear stresses by integration of the equilibrium equation, differences between both model will be found. Clearly all the profiles indicate a local bending, with almost identical values of tensiles and compression stresses, of each stiff layer "independent" one of the other. 

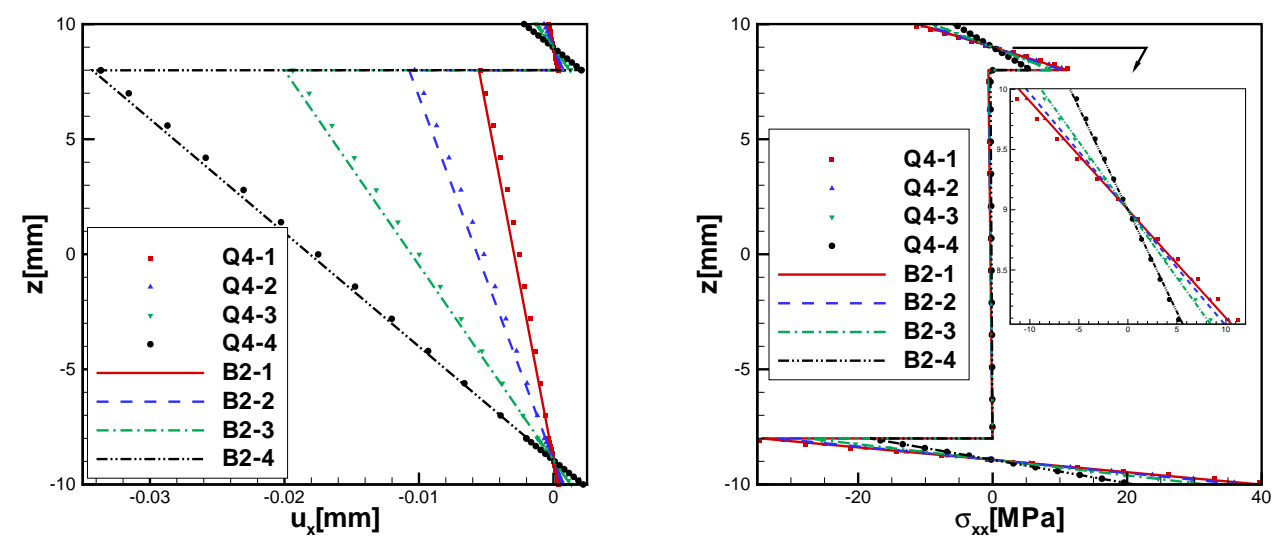

Figure 6: Profiles of horizontal displacements and axial stresses in the damaged beam

Figure 7.a shows the profiles of transverse shear stresses for the four mentioned sections obtained with the solid model and with the beam model by integration of the equilibrium equations from the axial stresses. Naturally shear stresses not only cancel out on the external surfaces but also on the weak layer. In the solid model it can be seen that the shearing force is mainly taken by the rigid layers with a low contribution of the core, that is even smaller in the vicinity of the support. The beam model in contrast predicts the same profile for all sections, so just one curve is included in the plot. This is because the RZT function $\phi$ has spent its potential in predicting the discontinuity and does not have the additional possibility to correctly approximate the distribution of axial stresses in the area near the fixed support. This is also seen in Figure 7.b where now the continuous component of the shear stress is practically zero (not shown) and only the part associated with the strain measure $\eta$ is relevant (just section 1 shown).

This also explains the larger stiffness of the beam model. In a section with a very weak layer the RZT leads to a practically constant shear strain across the thickness, just as it happens with the FSDT in an undamaged section where it is necessary to use a shear correction factor (SCF). The RZT does not use SCF, so in this case it is stiffer (4\%) than the solid model.

From these plots it can be concluded that for a section that includes a very weak layer:

- The RZT substantially improves the FSDT capturing the discontinuity, but it is stiffer than the solid model

- The shear stresses away from the kinematic restriction (fixed support) 


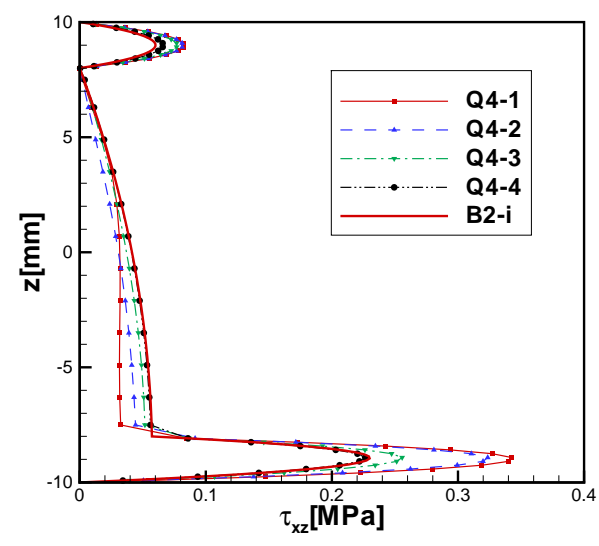

(a)

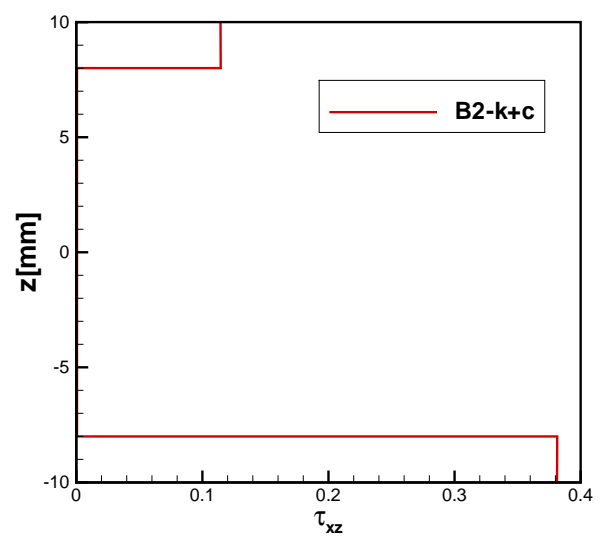

(b)

Figure 7: Transverse shear stress profiles in the damaged beam

can be accurately determined by integration of the equilibrium equation.

- The RZT predicts a constant profile of shear stresses along the beam, controlled exclusively by the strain measure $\eta$ and therefore discontinuous

In the paper by Groh and Tessler, a beam clamped at both ends with a normal traction load of sinusoidal variation and a shear traction load of cosinusoidal variation applied on the external surfaces with different value, whose resultant is an axial component and a moment is studied. The crosssection is asymmetric with a much weaker thin central layer (h/100). In this example, on one hand, the maximum relationship between modules is of the order of $10^{-3}$ (in this work is $10^{-9}$ ), on the other hand the geometric configuration (bi-clamped) and loads (variable shear) do not allow to observe the aspects described here.

\subsubsection{Partially damaged beam}

Finally we consider the case in which the first half of the beam is undamaged and that in the second half the union between the core and the upper layer is damaged. For the beam model the point of union of the two sections is critical. Three formulations to model the change of section in the beam are used, the two conforming models mentioned in the previous section and the non-conforming model. Figure 8. a shows the vertical displacement of the beam axis with the three beam models plus those obtained with a solid model used as reference results. It can be seen that the non-conforming model leads 


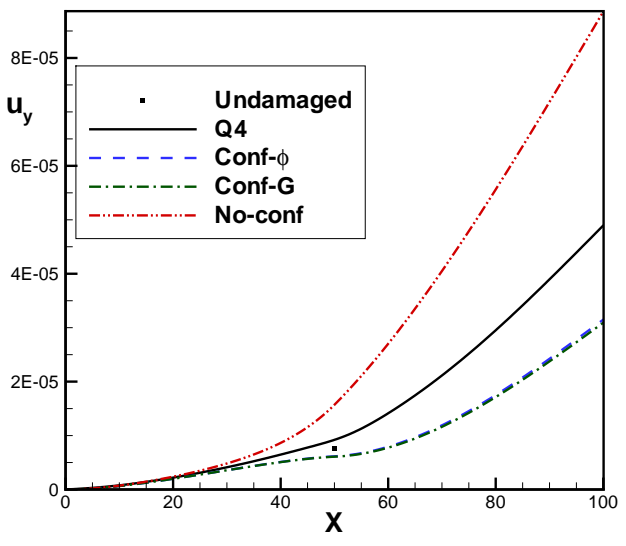

(a)

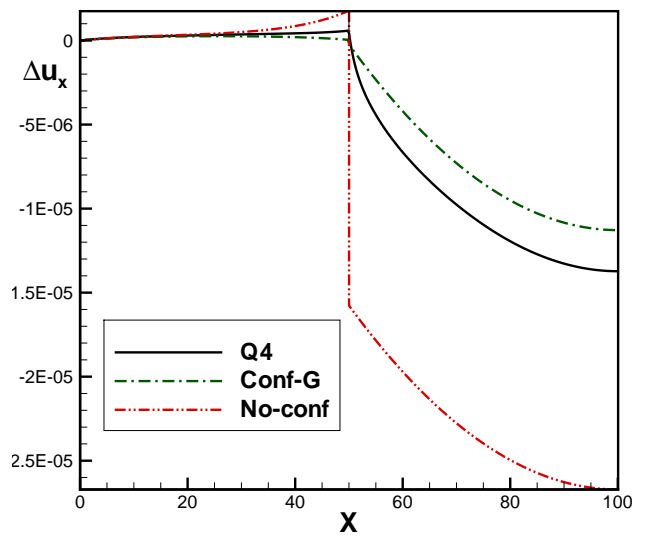

(b)

Figure 8: Partially damaged beam (a) transverse displacement of the axis (b) additional displacement in the upper border of the core

to unacceptable results, while the two conforming models give almost identical results and show a stiffer behavior than expected. This larger stiffness is associated with the important restriction imposed by the continuity of the additional variable $\psi$. The isolated point (reference "Undamaged") denotes the vertical displacement of the original undamaged beam axis at $x=\frac{L}{2}$. It can be seen in the reference solid model, that the damage of the second half implies a stress redistribution and a greater displacement at half of the beam than in the undamaged case. In contrast in the conforming beam models this displacement is smaller. Figure 8.b shows the additional displacement $\phi(z=8.005) \psi$ that occurs at the top border of the core. In the case of the reference model (Q4) this value is obtained by subtracting from the displacement of the point the weighted average of the bottom and top surface displacements of the section. The strong discontinuity that appears in the non-conforming model can be observed.

The other aspect that must be considered is that, in the case of a delamination process, on the delamination front or tip of crack there is a stress concentration that can hardly be captured with a beam model. It was shown above that for a discontinuity in the section the RZT leads, using kinematic equations, to a constant shear strain in the section and, using the constitutive equations, to constant shear stress in each layer. Figure 9 compares the stress profiles at two sections located symmetrically with respect to the section change, at a distance $d=\frac{L}{160}$. The references "Q" indicates results obtained with solid elements and "B" obtained with beam elements, while " $\mathrm{U}$ " (undamaged section) indicates the section to the left of the section change 


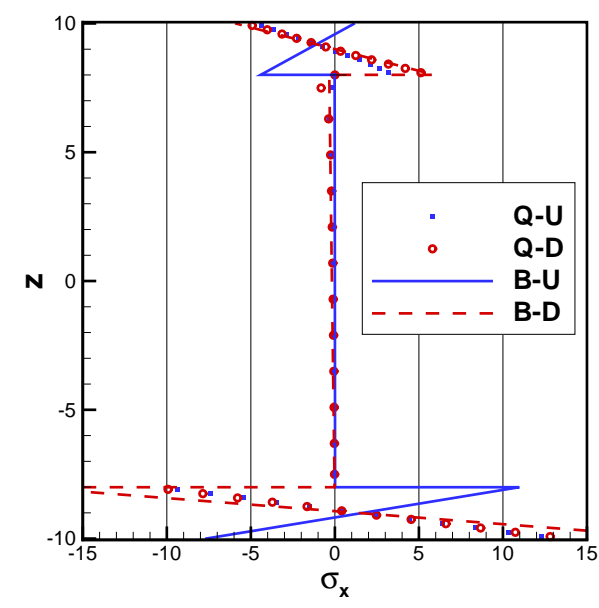

(a)

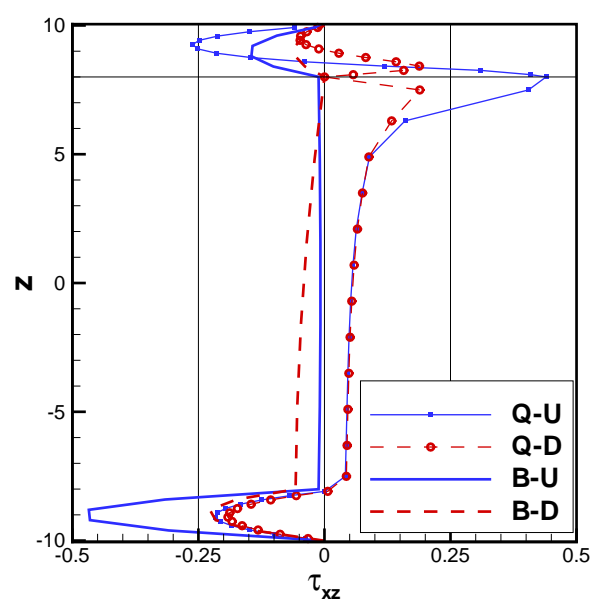

(b)

Figure 9: Partially damaged beam. Profiles of $\sigma_{x}$ and $\tau_{x z}$

and "D" (section Damaged) indicates the section to the right of the section change. Figure 9.a compares the profiles of axial stresses. It can be seen that the solid model has, as expected, a gradual change between one section and the other, while the beam model indicates extremely abrupt changes with inversion of the sign of the stresses. The results with the solid model are much more alike the fully damaged beam that can not transmit shear stresses between the lower and upper part. In the Figure 9.b the profiles of shear stresses are shown. Again, few changes between profiles in the solid model are seen, with a strong concentration (in the undamaged part) at the coordinate $z$ of the layer where damage exists. While in the beam model the shear stresses (obtained by integration of the equilibrium equations) show a parabolic distribution in each stiff layer and very low values in the core and is not able to predict the stress concentration present in the solid model.

In the work of Groh and Tessler the same beam above mentioned is studied, but now the thin weaker layer includes a partial delamination in $1 / 10$ of its length. The original elastic modulus of the material is roughly $1 / 10$ of the modulus of the rest of the layers in the laminate, while for the degraded material its elastic properties are reduced to $1 / 100$ of the pristine ones. The results presented are very good compared to the reference solution obtained with solid elements. However, the stress profiles do not correspond strictly to the points along the beam where section changes, so it can not be assured that in those points the stresses will be obtained accurately with the RZT. Besided, they mention modeling problems involved due to the discontinuity 
in cross sections and the need to employ strategies to handle it. For this reason, in this paper raises the requirement to enrich the formulation in order to be able to assess with some precision the stresses on the delamination front in order to establish a strategy to follow a delamination process.

\subsection{Buckling of a cylinder under axial load}

In this example, the behavior of a cylinder clamped at both ends and subjected to axial load is studied. The radius of the cylinder is $R=10 \mathrm{~m}$ and the total length $L=20 \mathrm{~m}$ while the thickness is $t=250 \mathrm{~mm}$ with a section defined by a symmetric sandwich laminate with the material properties indicated in the Table 2 and the stacking sequence in Table 3 where the principal direction of the laminate is the direction tangent to the parallel.

\begin{tabular}{|c|r|r|r|r|r|r|}
\hline Mat. & $E_{1}$ & $E_{2}$ & $E_{3}$ & $\nu_{12}, \nu_{13}$ & $\nu_{23}$ & $G_{12}, G_{13}, G_{13}$ \\
\hline \hline 1 & 50 & 10 & 10 & 0.05 & 0.25 & 5 \\
\hline 2 & 0.01 & 0.01 & 0.07585 & 0.01 & 0.01 & 0.0225 \\
\hline
\end{tabular}

Table 2: Material Properties $\left(E_{I}\right.$ and $G_{I J}$ en GPa) for buckling problem

\begin{tabular}{|c|r|r|}
\hline Mat. & Thick.[mm] & Orient.[degrees] \\
\hline 1 & 12.5 & 0 \\
\hline 1 & 12.5 & 90 \\
\hline 2 & 200.0 & 0 \\
\hline 1 & 12.5 & 90 \\
\hline 1 & 12.5 & 0 \\
\hline
\end{tabular}

Table 3: Laminate stacking sequence for buckling problem

Only the lower half of the cylinder has been modeled by imposing symmetry conditions at middle length. This arbitrarily restricts the bifurcation mode to such symmetry but this is irrelevant for the purposes of the comparison. The discretization with one-dimensional 2-node elements B2 includes meshes of 100, 200 and 400 elements, while meshes with two-dimensional elements Q4 include 20 elements in the thickness (6 in each outer layer and 8 for the core) and 100, 200, 400, 800, 1600 and 3200 divisions in the axial direction in order to obtain a converged solution for comparison.

Figure 10 shows the convergence in the critical load and the number of half waves as a function of the discretization in the axial direction. The shell of revolution element converges very fast and even with the coarsest discretization of 100 elements the results are very good. Also with the coarsest 


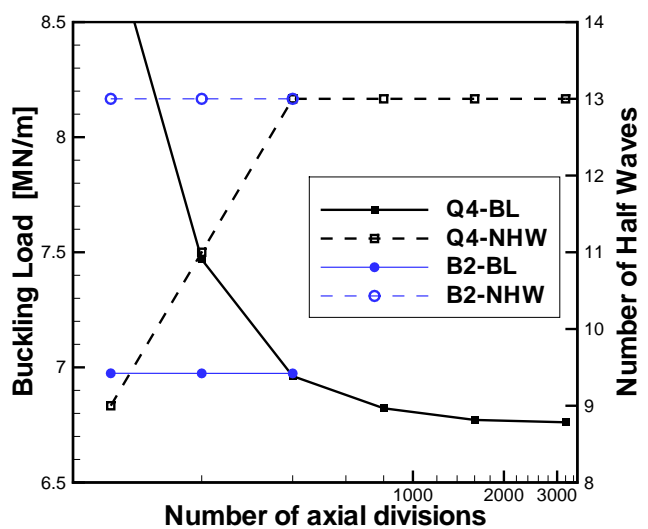

Figure 10: Convergence in buckling load and mode

discretization but with 50 3-noded elements, the same results as those obtained with the finest discretization using 2-node elements are found (6.974 $[\mathrm{MN} / \mathrm{m}]$ and 13 half waves). Besides, as expected, the two-dimensional element converges much more slowly but does to a lower critical load value $(6.761[\mathrm{MN} / \mathrm{m}])$ and the same number of half waves. This result shows three aspects:

- A well known one, which indicates that a solid model requires a very fine discretization (a very high number of elements) to obtain a converged solution. Notice that in this case the discretization is 2D and therefore easy to use, but that in general most of the problems require a 3D discretization.

- The shell model including the RZT converges much faster, that is, with discretizations that are 1 order of magnitude smaller in the length, and in addition is 1D, so that the amount of degrees of freedom involved is at least 2 orders of magnitude less.

- The FSDT + RZT model can not capture all the details of the behavior through thickness, which leads to slightly stiffer results but with values representative of the structural behavior.

Figure 11 shows the buckling mode of the cylinder for solid and shell models. The figure also includes the buckling mode that occurs in a cylinder where the inner layer and the core have been separated (delaminated) along $\frac{1}{20}$ of their length $L$ in the central zone (in this case due to the characteristics of the element in axisymmetric form, that is to say along all the parallel). 


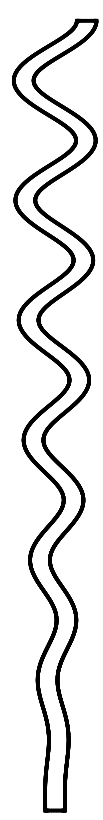

(a)

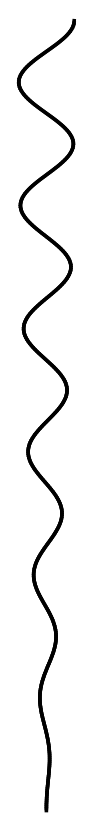

(b)

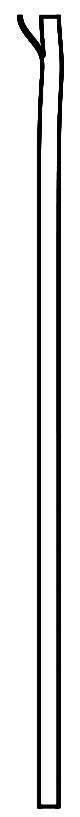

(c)

Figure 11: Buckling modes (a) Q4, (b) B2 (c) Q4 with a delamination of $L / 20$

The buckling mode in such case is local, which can not be captured by the shell model including the RZT which can only capture discontinuities in the displacements in the plane of the shell.

\section{Conclusions}

The objective of this paper is to study the influence of discontinuities of the cross section in the behavior of the refined zigzag theory (RZT). For this purpose, a two-dimensional shell element (curved beam and shell of revolution) and the basic aspects of the RZT are initially presented. Then an example with and without discontinuities is detailed analyzed. Besides the performance of the RZT in a buckling problem is studied. The conclusions that are obtained from the study for sandwich sections are:

- For beams without discontinuities in the section:

- The stress states obtained in the usual FEM way (kinematics plus constitutive equations) are very good in areas away from those points with kinematic restrictions (supports). 
- Even at such points the axial stresses are obtained with very good accuracy, which allows computing the shear stresses by integration in the transverse direction of the equation of equilibrium in the axial direction

- For beams with discontinuities in the section:

- The RZT allows to represent the discontinuity in the in-plane displacement (not in the normal direction).

- The RZT loses the ability to correctly represent the axial strain across the section, so that the precision in computing the shear stresses by integration and the bending stiffness decreases.

- The strain measure $\eta=\gamma-\psi$ is dominant, leading to a stiffer model than expected with uniform states of transverse strain and therefore discontinuous shear stresses.

- For beams with an abrupt change of the properties of the section (beam partially damaged):

- A "non-conforming" approach leads to inconsistent behavior.

- The conforming approaches introduce a significant restriction so the behavior becomes stiffer. Then in the case that is of particular interest here, i.e. when a thin layer has a very low modulus of elasticity associated with a degradation or damage process, none of the described conforming options leads to acceptable results.

- The axial stresses are not determined correctly in the discontinuity so they can not be used to obtain a reasonable approximation for shear stresses.

- It is not possible to capture the stress concentration that occurs in a discontinuity.

- Shell buckling

- The models with beam/shell elements converge rapidly and lead to very good results.

- For partially damaged sections it is not possible to predict that the results will be correct. 


\section{Acknowledgment}

First author acknowledges the financial support from CONICET (Argentina) and SeCyT-UNC. This work has also been supported by European Research Council through of Advanced Grant: ERC-2012-AdG 320815 COMP-DES-MAT "Advanced tools for computational design of engineering materials", by the Spanish Ministerio de Economía y Competividad through the projects: MAT2014-60647-R (OMMC)" and BIAS2015-67807-R (RESCICLO), and by International Center for Numerical Method in Engineering (CIMNE). All this support is gratefully acknowledged.

\section{References}

[1] A. Barut, E. Madenci, and A. Tessler. $C^{0}$-continuous triangular plate element for laminated composite and sandwich plates using the $\{2,2\}$ Refined Zigzag Theory. Composite Structures, 106:835-853, 2013.

[2] E. Carrera. Historical review of Zig-Zag theories for multilayered plates and shells. Applied Mechanics Reviews, 56:298-308, 2003.

[3] M. Di Sciuva, M. Gherlone, M. Iurlaro, and A. Tessler. A class of higherorder $C^{0}$ composite and sandwich beam elements based on the Refined Zigzag theory. Composite Structures, 132:784-803, 2015.

[4] A. Eijo, E. Oñate, and S.H. Oller. A four-noded quadrilateral element for composite laminated plates/shells using the refined zigzag theory. International Journal for Numerical Methods in Engineering, 95:631660, 2013.

[5] A. Eijo, E. Oñate, and S.H. Oller. A numerical model of delamination in compoosite laminated beams using the lrz beam element based on the refined zigzag theory. Composite Structures, 104:270-280, 2013.

[6] A. Eijo, E. Oñate, and S.H. Oller. Delamination in laminated plates using the 4-noded quadrilateral qlrz plate element based on the refined zigzag theory. Composite Structures, 108:456-471, 2014.

[7] F.G. Flores. Two-dimensional shell element for nonlinear analysis. Applied Mechanics Reviews, 48(11S):S30-S35, 1995.

[8] F.G. Flores. Implementation of the refined zigzag theory in shell elements with large displacements and rotations. Composite Structures, 118:560-570, 2014. 
[9] F.G. Flores, L.G. Nallim, and S.H. Oller. Formulation of solid-shell finite elements with large displacements considering different transverse shear strains approximations. Finite Elements in Analysis and Design, 130:39-52, 2017.

[10] M. Gherlone, A. Tessler, and Di Sciuva M. $C^{0}$ beam element based on the refined zigzag theory for multilayered composite and sandwich laminates. Composite Structures, 93:2882-2894, 2011.

[11] R.M.J. Groh and A. Tessler. Computationally efficient beam elements for accurate stresses in sandwich laminates and laminated composites with delaminations. Computer Methods in Applied Mechanics and Engineering, 320:369-395, 2017.

[12] L. Iurlaro, M. Gherlone, M. Di Sciuva, and Tessler. Assessment of the Refined Zigzag Theory for bending, vibration, and buckling of sandwich plates: a comparative study of different theories. Composite Structures, 106:777-792, 2013.

[13] L. Iurlaro, M. Gherlone, M. Di Sciuva, and A. Tessler. The (3,2)-Mixed Refined Zigzag Theory for generally laminated beams: Theoretical development and $C^{0}$ finite element formulation. Solids and Structures, 73-74:1-19, 2015.

[14] L.G. Nallim, S.H. Oller, Oñate E., and Flores F. A hierarchical finite element for composite laminated beams using a refined zigzag theory. Composite Structures, 163:168-184, 2017.

[15] E. Oñate, A. Eijo, and S.H. Oller. Simple and accurate two-noded beam element for composite laminated beams using a refined zigzag theory. Computer Methods in Applied Mechanics and Engineering, 213-216:362382, 2012.

[16] J.C. Simo and D.D. Fox. On a stress resultant geometrically exact shell model. part I: Formulation and optimal parametrization. Computer Methods in Applied Mechanics and Engineering, 72:267-304, 1989.

[17] A. Tessler. Refined zigzag theory for homogeneous, laminated composite, and sandwich beams derived from Reissner's mixed variational principle. Meccanica, 50:2621-2648, 2015.

[18] A. Tessler, M. Di Sciuva, and M. Gherlone. A consistent refinement of first-order shear deformation theory for laminated composite and sandwich plates using improved zigzag kinematics. Journal of Mechanics of Materials and Structures, 5:341-367, 2010. 
[19] A. Tessler, M. Di Sciuva, and M. Gherlone. A homogeneous limit methodology and refinements of computationally efficient zigzag theory for homogeneous, laminated composite, and sandwich plates. Numerical Methods for Partial Differential Equations, 27:208-229, 2011.

[20] D. Versino, M. Gherlone, and Di Sciuva M. Four-node shell element for doubly curved multilayered composites based on the the Refined Zigzag Theory. Composites Structures, 11B:392-402, 2014.

[21] D. Versino, M. Gherlone, M. Mattone, M. Di Sciuva, and A. Tessler. $C^{0}$ triangular elements based on the Refined Zigzag Theory for multilayered composite and sandwich plates. Composites Part B, Engineering, 44B:218-230, 2013. 